\title{
Agôn
}

Revue des arts de la scène

HS 2 | 2014

Mettre en scène le conte

\section{La rencontre de Cendrillon avec Blanche neige : décaler l'intrigue tout en respectant les enjeux fondamentaux du conte}

Entretien réalisé par Marion Boudier

François Flahault et Marion Boudier

\section{(2) OpenEdition}

Journals

Édition électronique

URL : http://journals.openedition.org/agon/3091

DOI : 10.4000/agon.3091

ISSN : 1961-8581

Éditeur

Association Agôn

Référence électronique

François Flahault et Marion Boudier, «La rencontre de Cendrillon avec Blanche neige : décaler

l'intrigue tout en respectant les enjeux fondamentaux du conte ", Agôn [En ligne], HS 2 | 2014, mis en ligne le 09 septembre 2014, consulté le 15 septembre 2020. URL : http://journals.openedition.org/ agon/3091

Ce document a été généré automatiquement le 15 septembre 2020

Association Agôn et les auteurs des articles 


\section{La rencontre de Cendrillon avec Blanche neige : décaler l'intrigue tout en respectant les enjeux fondamentaux du conte}

Entretien réalisé par Marion Boudier

François Flahault et Marion Boudier

1 Philosophe et anthropologue, François Flahault est directeur de recherche émérite au CNRS. Il travaille sur les contes et récits oraux depuis une trentaine d'années, refusant une approche par les symboles pour s'interroger sur les conceptions de l'homme que véhiculent ces histoires et leurs différentes versions. Il a notamment publié sur ce sujet La Pensée des contes (2001). Dans Be Yourself! Au-delà de la conception occidentale de l'individu (2006), il montre en quoi les contes sont porteurs d'une forme de savoir préphilosophique : en soulignant l'importance de la relation aux autres, ils invitent à penser l'identité comme relationnelle, évolutive et non comme une substance.

2 La lecture des essais de François Flahault a nourri une partie de la réflexion de Joël Pommerat ${ }^{1}$, notamment son ouvrage Le Sentiment d'exister ${ }^{2}$ au moment de l'écriture de Je tremble (1 et 2).

Marion Boudier - Pour aborder la question de l'adaptation des contes par Joël Pommerat, je vous propose que nous partions de votre ressenti de spectateur - un spectateur averti, spécialiste des contes.

François FLAHAULT - J'ai beaucoup apprécié la mise en scène de Cendrillon. Il y a une manière de se décaler par rapport à l'intrigue telle que tout le monde la connaît : le plaisir ne vient pas seulement du spectacle, mais du rapport entre le spectacle et ce qu'ils connaissent. Il y a toute une gamme d'affects déclenchés par ce jeu.

Ce qui m'a surtout frappé dans cette réécriture, c'est la façon dont Pommerat injecte dans le conte quelque chose qui n'y est pas, mais qui y entre très bien: la problématique de Blanche neige. Est-ce que la mère accepte ou non de passer le relais à sa fille ? Le conte repose sur le fait que la mère ne le passe pas. Dans Cendrillon de 
Pommerat, la belle-mère tient ce rôle. Ce qu'il y a de commun à Blanche neige, Cendrillon et beaucoup d'autres contes, c'est la situation problématique d'être tiraillé entre deux générations : lié à la génération de ses parents, mais devant faire sa place dans sa propre génération; c'est aussi la problématique de Peau d'âne : comment échapper à un lien incestueux avec son père et faire son chemin dans la vie. J'ai trouvé cela très présent dans le travail de Pommerat. Et la jalousie des sœurs pose la question de la coexistence, la difficulté de n'être qu'un parmi d'autres. On observe une double jalousie : d'une génération à l'autre et dans la même génération.

Pommerat problématise aussi le lien avec la mère morte de façon différente: Cendrillon se convainc qu'elle doit toujours penser à sa mère, et donc ne pas faire son deuil. C'est la même chose pour le prince. La nécessité de décrocher de la génération antérieure, de la mère, pour se raccrocher à sa propre génération est bien mis en valeur. Du coup, est laissé un peu de côté un élément présent dans d'autres versions du conte où la possibilité de devenir une femme est transmise par la mère : la fille ne devient pas femme par elle-même mais du fait d'une transmission par la mère. Ce geste de transmission implique que la mère s'efface - ce que la mère de Blanche neige ne fait pas. L'ambivalence des mères est souvent soulignée; elles ne veulent pas que leur fille les quitte, d'où une tension autour de la difficulté de la séparation; le blâme porte le plus souvent sur la mère ou sur les parents. Le jeune (le héros ou l'héroïne) est présenté comme innocent, exempt d'un attachement excessif à son père ou à sa mère. Les auditeurs du conte s'identifient au personnage principal, aussi, pour que le récit leur fasse plaisir, les sentiments qui peuvent être source de culpabilité doivent plutôt être imputés à d'autres personnages. Barbe bleue est l'un des rares contes où l'héroïne est présentée comme coupable puisqu'elle transgresse un interdit (mais elle l'est fort peu en comparaison de Barbe bleue, un serial killer). Dans les versions orales du Petit Chaperon rouge, également, l'héroïne est loin d'être aussi innocente que dans la version de Perrault : le loup lui propose en guise de collation des restes de sa grand-mère; un oiseau la met en garde, mais elle passe outre et mange son repas cannibale!

M. B. - Dans la Cendrillon de Pommerat, Sandra est à elle-même son propre opposant. C'est une figure d'héroïne coupable de son propre malheur.

F. F. - Oui, Pommerat a souligné un caractère problématique, beaucoup plus que chez Perrault. C'est une Cendrillon névrosée.

M. B. - La peur d'être abandonnée devient la peur d'abandonner sa mère.

F. F. - C'est comme si elle devait réparer sa mère post mortem en pensant tout le temps à elle. C'est une attitude d'auto mortification; elle se fait souffrir. Ce n'est pas seulement sa belle-mère qui la fait souffrir: c'est un ajout spécifique fait par Pommerat, qui rend le spectacle quelque peu grinçant. On perd une forme de grâce qui compensait la noirceur. Et le prince n'est pas terrible non plus! Mais c'est drôle. Pommerat est quand même très noir ; c'est du moins l'impression que j'ai eue lorsque nous avions échangé à propos de mon livre Le Sentiment d'exister.

M. B. - Dans ce livre, vous consacrez des pages à l'idéalisation et à la « désidéalisation » de soi, qui ont en partie influencé l'écriture de certaines scènes du Présentateur de Je tremble ( 1 et 2) et auxquelles le personnage de Sandra peut aussi faire écho. Sandra n'est pas dans l'idéalisation mais dans le dénigrement d'elle-même, ce qui revient au même problème d'image et de perception. Elle a une perception biaisée de sa mère et cela fausse son rapport au réel. C'est un type de personnage 
qu'on trouve dans d'autres pièces de Pommerat, des personnages qui ont des conflits de perception, qui mêlent plusieurs réalités et qui, précisément, ont du mal à se sentir exister. Cette Cendrillon est réécrite selon un axe fort, qui est extériorisé, alors que les personnages de conte, il me semble, sont d'ordinaires plus ouverts à plusieurs interprétations : Sandra est-elle donc encore un personnage de conte?

F. F. - On n'est plus dans le conte populaire, car l'objet est plus complexe, avec, de surcroît, un côté parodique. Certains contes touchent à la question du deuil. Dans les versions traditionnelles de Cendrillon, l'héroïne a déjà fait son deuil : elle garde un lien avec sa mère, mais en ayant accepté qu'elle soit morte. Par exemple, dans une version vietnamienne, elle s'adresse à un petit poisson qu'elle a dans un bassin : c'est une sorte de prière ; le poisson représente la bienveillance de sa mère. Ce que fait Pommerat, en représentant plusieurs personnages (Sandra, le prince) qui n'arrivent pas à faire leur deuil, est très particulier. Ils y arrivent in extremis lorsque Sandra dit au prince que sa mère est morte et accepte d'avoir aussi perdu la sienne. Pourquoi pas ? Je trouve que ça marche très bien!

M. B. - Dans certaines versions, Cendrillon trouve la robe de sa mère sur sa tombe. Dans le conte de Pommerat, elle va au bal en portant une robe que sa mère avait portée pour un mariage.

F. F. - Oui, j'étais très satisfait en voyant le spectacle, car tout en ayant modifié certains motifs, Pommerat n'en fait pas moins sentir les enjeux fondamentaux de I'histoire. II a inventé beaucoup de choses mais il n'a absolument pas dénaturé l'affaire. Une question que je me suis posée, étant donné que le livre est publié dans une collection jeunesse, c'est la destination du spectacle : s'adresse-t-il à un jeune public?

M. B. - Non, c'est un spectacle tout public à partir de 8 ans. Comme il l'a expliqué dans des entretiens, Pommerat n'adhère pas trop à l'appellation « théâtre jeune public » : il ne change pas sa manière de travailler pour les contes.

F. F. - Oui, j'ai apprécié que ça ne fasse pas « spectacle pour enfants ». C'est peut-être un peu grinçant, un peu noir pour des enfants, car les personnages qui sont censés être positifs ne le sont pas vraiment. Cendrillon névrosée qui se fait du mal, c'est peut-être difficile pour des plus jeunes.

M. B. - L'histoire fınit bien, mais en effet, à qui s'identifier? A la fée peut-être?

F. F. - La fée est caricaturée dans cette version. Elle est ridicule, foireuse, ce qui est comique, mais elle ne sert à rien. Elle n'apparaît pas comme un substitut de la mère, ce qu'elle est chez Perrault où elle personnifie la bienfaisance de la mère morte. Dans les versions populaires, il n'y a pas de fée, mais des vêtements transmis d'outretombe, grâce auxquels la bienfaisance maternelle continue à s'exercer. C'est un aspect très touchant de ce type de contes: des personnages en état de disgrâce, de relégation, qui ne trouvent pas leur place, se tournent vers une puissance invisible, laquelle leur vient en aide. Ce n'est jamais présenté dans les contes comme un thème religieux. On a plutôt là affaire à quelque chose de protoreligieux.

M. B. - Trouver sa place, c'est une grande question des contes.

F. F. - Oui. Exister c'est avoir une place parmi les autres, une place dans la société, être situé dans une parenté et un lignage. Personne n'existe par soi-même : les contes pour moi ont été une sorte d'antidote par rapport à l'idéologie occidentale moderne du self. On peut rapprocher l'histoire de Cendrillon de la fameuse phrase de Simone de Beauvoir : «on ne naît pas femme on le devient ». Beauvoir présuppose que c'est soit la société qui l'impose, soit la femme qui le décide par elle-même. Mais le conte nous dit autre chose : oui, on ne naît pas femme, on le devient, mais on le devient à condition que la mère transmette à la fille ce don de devenir femme et lui laisse la 
place. C'est bien vu; les psychologues cliniciens rencontrent des cas de jeunes femmes qui n'arrivent pas à avoir d'enfant alors que physiologiquement tout va bien ; c'est leur relation avec leur mère qui bloque. La question de la transmission est essentielle: la clinique le montre, le conte le dit, mais cela semble étranger à l'idéologie moderne.

M. B. - Dans son analyse du conte, Bettelheim évoque cette question de la transmission entre femmes : la mère, ou la belle-mère, doit s'effacer pour laisser la place à la jeune fille, pour que celle-ci devienne femme et mère à son tour. Mais la question de la sexualité est laissée de côté par Pommerat qui a fait des personnages de très jeunes gens.

F. F. - Oui, sauf dans la scène des deux sœurs avec le mannequin. Mais la sexualité est montrée plutôt sous un jour défavorable. Trouver un équilibre entre noirceur et bienveillance est chose délicate. Fanny et Alexandre de Bergman me semble un bon exemple de cet équilibre entre noirceur et bonté. Son dernier film est très beau aussi : Saraband, avec en filigrane Peau d'âne.

M. B. - Par rapport au self et à cette question d'équilibre ou de nuances dans le traitement, je trouve le personnage de la belle-mère intéressant : cette marâtre peut aussi susciter de l'empathie ou de la pitié, car elle vit une désillusion (elle croyait au prince charmant). Est-ce qu'on trouve ce genre de personnage complexe dans d'autres versions du conte?

F. F. - Elle a en effet un côté Bovary. Je ne connais pas de version qui nuancent la belle-mère de cette manière, sauf dans les contes où il n'y a pas deux personnages opposés (mère/ belle-mère) mais un seul : une mère ambivalente.

M. B. - Avec la belle-mère, Pommerat déplace aussi le motif de la chaussure.

F. F. - C'est un déplacement que j'ai trouvé amusant. Mais pour l'héroïne et le jeune prince, la chaussure joue bien son rôle d'identification, de "carte de visite ", ce qui est fidèle à l'esprit du conte. De nombreux contes mettent scène deux composantes de ce qui lie entre eux deux personnages. D'abord la puissance de ce que Lacan appelait l'imaginaire : l'attrait exercé par l'éclat, la beauté, la parure. Et puis, parce que cela ne suffit pas, l'objet tiers qui certifie que c'est bien lui, c'est bien elle : en l'occurrence, la chaussure qui fait preuve: une relation humaine ne peut pas fonctionner seulement sur l'image, il faut aussi être identifié par une marque dans un groupe. Les deux chaussures font la paire, c'est le symbolon.

M. B. - Nous n'avons pas encore parlé de la narratrice...

F. F. - On entend une voix avec un fort accent. C'est bien, c'est une histoire qu'on vous raconte. La facticité de la situation n'est pas dissimulée mais assumée. La présence de la voix, sans mise en scène, est essentielle dans les contes : ce qui est très important, c'est la relation à la voix humaine, la puissance de la voix humaine que je relie aussi au protoreligieux. Ce qui est parfois mis en scène dans les contes, c'est une allusion à la situation même de contage où la voix humaine joue un rôle de liant, de transmission de vie. Ce n'est pas une parole sacramentelle, mais c'est une parole qui a un pouvoir incroyable, une parole humaine qui se justifie par un bien culturel qu'elle transmet. Cela renvoie en même temps à l'origine de la vie : la voix de la mère que l'enfant entend dès avant sa naissance. Cette voix, physiquement présente, transmet un motif culturel, un chant, une comptine. 


\section{B. - On entend une voix féminine et on voit un conteur marcheur.}

F. F. - Le conteur est un médiateur entre l'histoire et l'auditoire. Il faut qu'il soit fortement présent pour transmettre, par la voix d'abord plus que par le corps. Le conteur n'est pas un acteur. Il parle en son nom, il ne joue pas un rôle.

J'ai récemment entendu un conte dit par une actrice avec un jeu d'acteur, j'ai trouvé que ça ne marchait pas bien. Prenons l'exemple des one man show : la personne qui est en scène joue, mais en même temps, elle est elle-même, elle ne s'efface pas derrière un rôle ou un personnage. Autre exemple d'un art de la parole : les bonimenteurs sur les marchés ; ce qu'ils racontent n'a pas d'intérêt, ils disent délibérément n'importe quoi, et pourtant, les badauds restent là à les écouter, ils sont pris par une sorte de magie verbale. Puissance pure de la parole ludique, avec un contenu minimal. Dans le conte, il y a un contenu bien sûr, mais il y a aussi cet art de la parole proche de celui du camelot. J'ai plusieurs fois écouté des conteurs étrangers dont je ne connaissais pas la langue, mais j'étais captivé, captivé par la consistance et la saveur de la parole elle-même. Il est fréquent que les contes donnent eux-mêmes matière à faire valoir la parole dans son rythme et ses sonorités grâce à des formulettes qui étaient psalmodiées ou chantées, qui se répétaient au cours de l'histoire.

M. B. - La situation de conter, la relation qu'elle implique serait donc plus importante que le contenu raconté ? Et la morale?

F. F. - Pas plus importante, mais complémentaire, un peu comme dans une liturgie. Est-ce qu'on peut être bien ensemble ou est-ce que les relations humaines sont déchirantes ? Dans son contenu, le conte dramatise cette tension entre la possibilité de coexister et son impossibilité. Et en même temps, dans la situation de contage, le conteur fait vivre aux auditeurs la résolution de cette tension. Le contenu du conte et la situation de contage se font mutuellement écho. Il faut être en adéquation avec les gens pour qu'ils passent un bon moment. Ce plaisir vécu est fondamental, le conte ne raconte pas pour transmettre une morale ou un enseignement. Italo Calvino a composé un important recueil de contes populaires italiens. Dans sa préface, il se demande s'il y a une morale dans les contes. Il répond par la négative. S'il y a quelque chose de moral dans les contes, écrit-il, c'est la situation de contage elle-même, c'est l'expérience vécue par ceux et celles qui sont là. Les conteurs cherchent avant tout à ce que les gens passent un bon moment. La morale liée au contenu a été ajoutée par Perrault, puis au XVIII ${ }^{\mathrm{e}}$ avec la naissance d'une littérature pour enfants à visée édifiante.

M. B. - La narratrice est sans doute Sandra très âgée; elle raconte donc sa propre histoire. Cette situation existe-t-elle dans les contes traditionnels?

F. F. - Non, dans les situations de contage traditionnel le conteur est extérieur à l'histoire. Je précise que ce sont les illustrateurs, à partir du XIX siècle (notamment Gustave Doré), qui ont situé les contes dans un lointain passé. Dans les situations de contage traditionnel, lorsque le conteur dit «il était une fois ", il se référait à un passé proche. Il lui arrivait de situer l'action dans des lieux connus des personnes auxquelles il s'adressait, leur permettant ainsi de les imaginer et de réagir.

Mai 2013 - Février 2014. 


\section{NOTES}

1. Voir Joëlle Gayot et Joël Pommerat, Joël Pommerat, troubles, Arles, Actes Sud, 2009, p. 53.

2. Éditions Descartes \& Cie, 2e édition, 2013. Extraits disponibles sur le site de l'auteur: francoisflahault.fr. La Pensée des contes peut également être téléchargé gratuitement à partir de ce site.

INDEX

Mots-clés : Pommerat (Joël), Cendrillon, conte 